\title{
A viewpoint for permutations with a low density of patterns
}

\author{
BenJAMin FinEMAN
}

\begin{abstract}
Analyzing block partitions of permutation matrices has proven useful in studying permutations with a low density of patterns. Conditioning on the size and density of various blocks provides a large amount of control on both the number and type of patterns that can exist globally in a permutation. Using this technique, we provide a bound for the number of permutations with a low density of patterns, and a strengthening of the pattern removal lemma in a similar vein to to Szemerédi's removal lemma for graphs. The term "low density" refers to permutations in $S_{n}$ containing fewer than $(\delta n)^{k}$ copies of a specified pattern of length $k$, for some $\delta>0$. When $n$ is sufficiently large, and $\delta$ is small, the number of these permutations, which we denote by $\chi_{\delta}^{n}(\gamma)$, satisfies
\end{abstract}

$$
a^{n} n ! \leq \chi_{\delta}^{n}(\gamma) \leq b^{n} n !
$$

where $a$ and $b$ only depend on $\delta$ and $k$.

AMS 2000 SUBJECT CLASSIFICATIONS: Primary 05A05; secondary 05D05.

\section{Introduction}

Unless otherwise stated, we use the "one-line notation" for permutations. A permutation $\sigma \in S_{n}$ will be written as $\sigma_{1} \sigma_{2} \ldots \sigma_{n}$, where $\sigma_{i}=\sigma(i)$ for $1 \leq i \leq n$. Suppose $\gamma \in S_{k}$, with $k \leq n$. Informally, an occurrence of the pattern $\gamma$ in $\sigma$ is a subsequence of $\sigma$ that is order-isomorphic to $\gamma$. Let $[n]=\{1,2, \ldots, n\}$. A permutation $\sigma \in S_{n}$ can be viewed as an element $\sigma=\left(\sigma_{1}, \ldots, \sigma_{n}\right) \in[n]^{n}$. We provide formal definitions in this more general setting with the aim of increasing clarity and precision in subsequent arguments. For $s \in[n]^{r}$ we use the notation $s(i)$ to denote the $i$-th element in the sequence $s$.

Definition 1.1. Let $s \in[n]^{r}$ and $k \leq r$. Define a map also called $s:[n]^{k} \rightarrow$ $[n]^{k}$ by $s(z)=(s(z(1)), s(z(2)), \ldots, s(z(k)))$. 
Definition 1.2. Let $g \in[n]^{k}$ and $s \in[n]^{r}$, with $k \leq r$. Define a map $J_{g}:[n]^{r} \rightarrow P\left([n]^{k}\right)$, where $P$ is the power set, by

$$
\begin{gathered}
J_{g}(s)=\{s(z) \mid z(i)<z(j) \text { and } s(z(i))<s(z(j)) \Leftrightarrow g(i)<g(j), \\
\text { for } 1 \leq i<j \leq k\} .
\end{gathered}
$$

Each $s(z)$ in $J_{g}(s)$ is called an instance, copy, or occurrence of the pattern $g$ in $s$.

For example, the permutation $1432 \in S_{4}$ contains three instances of the pattern 132, namely 143,142, and 132. If $\sigma$ contains no copies of $\gamma$, we say that $\sigma$ avoids $\gamma$.

The subject of pattern avoidance in permutations has been well studied (see, e.g., [2], [8], and [10]). Some areas of interest include enumerating and finding bounds for the number of permutations that avoid designated patterns or generalized patterns, as well as examining their asymptotics. Ultimately, the important question of what properties make certain patterns easier or harder to avoid is still unknown.

The most sweeping result pertaining to pattern avoidance is the StanleyWilf conjecture, proved by Marcus and Tardos [9].

Theorem 1.3 (Stanley-Wilf conjecture, 1980). Let $\gamma$ be any pattern, and let $\left|S_{n}(\gamma)\right|$ be the number of permutations in $S_{n}$ that avoid $\gamma$. Then there exists a constant $c$ so that for all positive integers, we have $\left|S_{n}(\gamma)\right| \leq c^{n}$.

The goals of this paper are twofold. First, we provide a result similar to the Stanley-Wilf conjecture, but for permutations with a low density of a certain pattern. Second, we give a strengthening of the pattern removal lemma for permutations discussed by Cooper [3].

Both arguments are similar in nature: we form block partitions of the permutation matrices and use geometric properties of the individual blocks to control the number of copies of a given pattern that can exist globally. We state the main theorems here but defer their proofs to later sections, after introducing the block partition matrices and providing some insight to their utility.

Theorem 1.4. Let $\gamma \in S_{k}$, and let $\chi_{\delta}^{n}(\gamma)$ be the number of permutations in $S_{n}$ with fewer than $\delta^{k} n^{k}$ instances of $\gamma$.

For every $k, \delta<1 /(2 k)$, there are $N$, and $a \leq b<1$, such that for $n>N$, we have

$$
\left(a^{n}\right) n ! \leq \chi_{\delta}^{n}(\gamma) \leq\left(b^{n}\right) n !
$$


In particular, we have $a=\delta^{k} / 2$, and $b=\left(\frac{e}{(k-1) \delta}\right)^{\delta}\left(\frac{k-1}{k}\right)^{1 / k}+t$ for any $t>0$, where $e$ is Euler's number.

Cooper, Lundberg, and Nagle [4] have independently reached a similar result that applies to generalized patterns. However, their constant $b$ converges to 1 at a much faster rate, as $k$ tends to infinity.

Note that the upper bound we have is indeed nontrivial. The term $\left(\frac{e}{(k-1) \delta}\right)^{\delta}$ approaches 1 , as $\delta \rightarrow 0$, and the second term $\left(\frac{k-1}{k}\right)^{1 / k}$ is a constant smaller than 1, depending only on the length of the permutation $\gamma$. Furthermore, we can choose $t$ and $\delta$ small enough, so that the quantity

$$
\left(\frac{e}{(k-1) \delta}\right)^{\delta}\left(\frac{k-1}{k}\right)^{1 / k}+t
$$

is strictly smaller than 1, giving a nontrivial bound. In particular, for a pattern of length 3 , the theorem above, with choice of $\delta=.001$ and $t=.01$, implies that for $n$ sufficiently large, there are at most

$$
n !\left\{\left(\frac{e}{(2)(.001)}\right)^{.001}\left(\frac{2}{3}\right)^{1 / 3}+.01\right\}^{n}<n !(.9)^{n}
$$

permutations with fewer than $(.001 n)^{3}$ instances of the pattern 132 .

Our second result characterizes permutations that have a low density of a certain pattern, in the same way that Szemerédi's graph removal lemma characterizes graphs with a low density of a certain subgraph; by showing that all instances of the pattern (or subgraph) can be "covered" by a small number of edges. In the case of graphs, removing those edges destroys all copies of the subgraph.

To state our second result, we adapt the notion of edges to permutations.

Definition 1.5. Define a map $E:[n]^{r} \rightarrow P\left([n]^{2}\right)$ by

$$
E(s)=\{(s(i), s(j)) \mid i<j\}
$$

$E(s)$ is called the set of edges of $s$.

Definition 1.6. A subset $E$ of $[n]^{2}$ covers a subset $J$ of $[n]^{r}$ if $E(s) \cap E \neq \emptyset$ for all $s \in J$.

Continuing our example with $\sigma=1432$ and $\gamma=132$, the set of edges of 1423 is given by

$$
E(1432)=\{(1,4),(1,3),(1,2),(4,3),(4,2),(3,2)\}
$$


$E(1432)$ trivially covers $J_{132}(1432)=\{143,142,132\}$, but so does the subset of edges $E=\{(1,4),(3,2)\}$. While not unique, $E$ is a subset of minimal size with this property.

Definition 1.7. Let

$$
d(s, g)=\min \left\{|E| / n^{2} \mid E \text { covers } J_{g}(s)\right\} .
$$

A permutation $\sigma$ is $\epsilon$-far from avoiding $\gamma$ if $d(\sigma, \gamma) \geq \epsilon$.

From the discussion above, it can be seen that $d(1432,132)=1 / 8$.

Theorem 1.8. Let $\gamma$ be a pattern of length $k$, and $\sigma \in S_{n}$. For $n$ sufficiently large, if $\sigma$ is $\epsilon$-far from avoiding $\gamma$, then the number of instances of $\gamma$ in the permutation $\sigma$ is at least $(\delta n)^{k}$, where

$$
\delta=\frac{\epsilon^{4}}{16} .
$$

Cooper [3] used his permutation regularity lemma to achieve a similar result, but with a different value for $\delta$. This result can also be obtained by using the graph regularity lemma on a $k$-partite graph, where the cardinality of each partite set is $n$, and edges between different sets are determined by both the permutation and pattern in question, so there is a bijection between instances of $\gamma$ in the permutation and copies of $K_{k}$ in the graph. We omit the details. The downside to both of these methods lies in the bounds: $\delta$ must be very small compared to $\epsilon$. Both versions rely on first applying Szemerédi's regularity lemma to obtain an $\epsilon$-regular partition, and then working with these sets, the size of which could be very small. Indeed, the number of sets in the partition only has a tower type upper bound (in the size of $1 / \epsilon$ ). Gowers [7] proves that this type of bound is indeed necessary to obtain regularity, by creating a graph whose vertices must be partitioned into that many sets in order to create an $\epsilon$-regular partition. It would be interesting to see if the same were true in the context of permutations, with respect to Cooper's version of regularity, i.e., if there is a permutation that is equally hard to regularize. We provide a proof where $\delta$ and $\epsilon$ have a polynomial relationship, giving a much stronger result.

Our interest in studying permutations with a low density of a certain pattern comes from Szemerédi's regularity lemma on graphs, and its accompanying removal lemmas. A good overview is contained in [1] and [5]. Understanding the structure of graphs that contain a low density of triangles (or any other subgraph) was the key to proving several results, including Szemerédi's Theorem [11] that sets of positive upper density contain arithmetic patterns of arbitrary length. Generalizing the results to hypergraphs 
[12] and proving a "sparse" version of Szemerédi's regularity lemma led to the celebrated result of Green and Tao [6] that the prime numbers contain arbitrarily long arithmetic progressions as well.

\section{Block partitions of permutation matrices}

As is customary in this field, when describing permutation matrices we use a coordinate system to describe the various entries of the matrix. Thus, the entry $(i, j)$ refers to the entry in the $i$-th column from the left, and $j$-th row from the bottom.

Definition 2.1. Given $\sigma=\sigma_{1} \sigma_{2} \cdots \sigma_{n}$, the permutation matrix $M_{\sigma}$ is an $n \times n$ binary matrix with $M_{\sigma}(i, j)=1$ if and only if $\sigma(i)=j$.

When it is clear that we are referring to the entries of the permutation matrix, we use the notation $\sigma_{i}$ for the entry $\left(i, \sigma_{i}\right)$ in the matrix $M_{\sigma}$. Thus $M_{\sigma}$ has a 1 at the positions $\sigma_{1}, \ldots, \sigma_{n}$, and a 0 at all other positions. The permutation $352987461 \in S_{9}$ is represented as:

the block matrix $M_{352987461}^{3}$

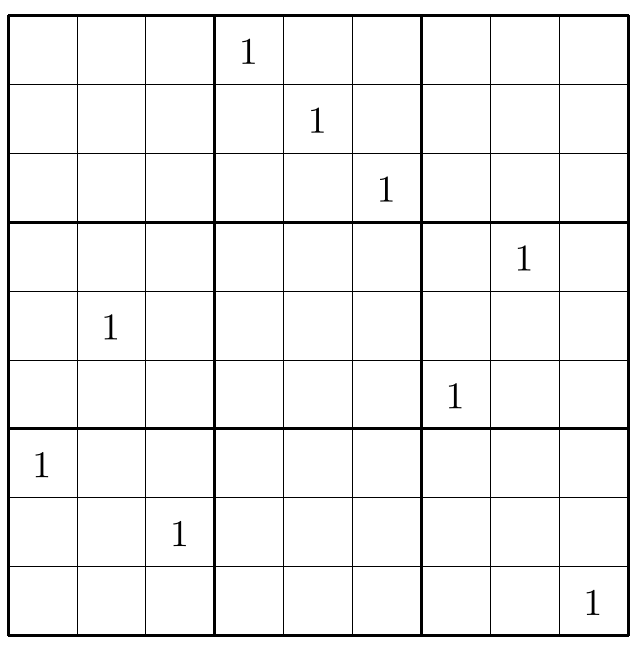

In the interest of brevity, we only show the positions of the 1's. Now, consider the $m \times m$ block partitioned matrix $M_{\sigma}^{m}$ formed from $M_{\sigma}$ by placing lines directly to the right of the $\lceil j n / m\rceil$-th column, and directly above the $\lceil j n / m\rceil$-th row, for $1 \leq j \leq m$. We use a coordinate system to refer to the different blocks. Continuing our example, the block matrix $M_{352987461}^{3}$ has nine blocks, each of which is a $3 \times 3$ binary matrix containing between zero and three 1's. 
The upshot is that there are collections of blocks which can force the existence of copies of $\gamma$ in the permutation. In the example, consider the blocks $B_{1,1}, B_{2,3}$, and $B_{3,2}$; any sequence $\left(\sigma_{x_{1}}, \sigma_{x_{2}}, \sigma_{x_{3}}\right)$ with $\sigma_{x_{1}} \in B_{1,1}$, $\sigma_{x_{2}} \in B_{2,3}$, and $\sigma_{x_{3}} \in B_{3,2}$ must be an instance of 132. In the example above, there are $(2)(3)(2)=12$ patterns that can be formed this way. If the size of the blocks we use is large, we can create a class of permutations with a large number of copies of a given pattern, and if we use blocks that are small enough, we can ensure that most patterns that occur in a permutation are of this type (entries from blocks in distinct rows and columns). These are the main ideas respectively behind our two results. We make this concept more precise below.

Definition 2.2. Let $z \in[n]^{k}$ and $B=\left(B_{1}, \ldots, B_{k}\right)$ be a sequence of blocks each in a distinct row and column, ordered from left to right. We say that $z$ is incident with $B$ if the entry $\sigma_{z(i)} \in B_{i}$ for all $i$.

Lemma 2.3. Let $z$ be incident with $\left(B_{x_{i}, y_{i}}\right)$, and let $\gamma \in S_{k}$. If

$$
y_{i}<y_{j} \Leftrightarrow \gamma(i) \leq \gamma(j)
$$

then $\sigma(z)$ is an instance of $\gamma$.

Proof. By construction, $\sigma(z(i))<\sigma(z(j))$ if and only if $\gamma(i)<\gamma(j)$, implying the claim.

\section{Bounds for the number of permutations with a low density of patterns}

We begin with the upper bound.

Definition 3.1. The order of the block $B_{x, y}$ is given by

$$
\left|B_{x, y}\right|=\left|\left\{i \mid \sigma_{i} \in B_{x, y}\right\}\right|
$$

which counts the number of 1 's in $B_{x, y}$.

Unless otherwise stated, let $n$ and $k$ be fixed, and assume $n$ is divisible by $k$. If $n$ is not divisible by $k$, the following arguments still hold after making some small technical adjustments.

Lemma 3.2. Let $\left(B_{x_{i}, y_{i}}\right)$ satisfy the assumptions of Lemma 2.3. If in addition,

$$
\min \left\{\left|B_{x_{i}, y_{i}}\right|\right\} \geq \delta n,
$$

then $\sigma$ contains at least $\delta^{k} n^{k}$ instances of $\gamma$. 
The contrapositive gives us a one-sided test, which can be used to give an upper bound for the number of permutations with fewer than $\delta^{k} n^{k}$ instances of $\gamma$.

Lemma 3.3. If $\sigma$ has fewer than $\delta^{k} n^{k}$ instances of $\gamma$, then $\left|B_{x_{i}, y_{i}}\right|<\delta n$ for some $i$.

Since the set of permutations with fewer than $\delta^{k} n^{k}$ instances of $\gamma$ must be a subset of the set of permutations in which one of the above blocks contains fewer than $\delta n$ 1's, finding an upper bound for number of permutations in the latter set also gives an upper bound for the number of permutations in the former.

Define for each $n$, a finite probability space $\left(\Omega, 2^{\Omega}, P\right)$, where $\Omega=\left\{M_{\sigma}^{k} \mid\right.$ $\left.\sigma \in S_{n}\right\}$ and $P$ is the uniform probability measure.

Lemma 3.4. For $\delta<1 /(2 k)$ and $n$ sufficiently large, there exists $c$ such that the probability that $\min \left\{\left|B_{1, \gamma_{1}}\right|,\left|B_{2, \gamma_{2}}\right|, \cdots,\left|B_{k, \gamma_{k}}\right|\right\}<\delta$ is at most

$$
c n\left[\left(\frac{e}{(k-1) \delta}\right)^{\delta}\left(\frac{k-1}{k}\right)^{1 / k}\right]^{n} \text {. }
$$

In the following proof we use the notation $(n)_{k}=(n)(n-1) \ldots(n-k+1)$ for the falling factorial. It can be seen that $\frac{(m)_{k}}{(n)_{k}} \leq(m / n)^{k}$ if $k<m<n$. Furthermore, we use the well-known fact that $\left(\begin{array}{l}n \\ k\end{array}\right) \leq\left(\frac{e n}{k}\right)^{k}$. To see this, note that $e^{k}=\sum_{i} k^{i} / i !>k^{k} / k !$, hence, $e^{k} k ! / k^{k}>1$. Thus,

$$
\left(\begin{array}{l}
n \\
k
\end{array}\right) \leq \frac{n^{k}}{k !}<\frac{n^{k}}{k !}\left(\frac{e^{k} k !}{k^{k}}\right)=\left(\frac{e n}{k}\right)^{k} .
$$

Proof. As a first step, we compute the probability that $\left|B_{1, \gamma_{1}}\right|<\delta n$. Multiplying this probability by $k$ gives an upper bound for the quantity in question.

Consider the first column of blocks $\bigcup_{i=1}^{k} B_{1, i}$. Note that $\sum_{i=1}^{k}\left|B_{1, i}\right|=$ $n / k$ because the permutation matrix $M_{\sigma}$ has a 1 in each column. Thus, if $\left|B_{1, a_{1}}\right|=j$ there must be $n / k-j 1$ 's in the blocks directly above it. The probability that $B_{1, a_{1}}$ contains at most $\delta n 1$ 's is given by:

$$
\begin{aligned}
& \frac{1}{\left(\begin{array}{c}
n \\
n / k
\end{array}\right)} \sum_{j=0}^{\delta n}\left(\begin{array}{c}
n / k \\
j
\end{array}\right)\left(\begin{array}{c}
(k-1) n / k \\
n / k-j
\end{array}\right) \\
& \quad=\frac{\left(\begin{array}{c}
(k-1) n / k \\
n / k
\end{array}\right)}{\left(\begin{array}{c}
n \\
n / k
\end{array}\right)}+\sum_{j=1}^{\delta n}\left(\begin{array}{c}
n / k \\
j
\end{array}\right)\left(\frac{(n / k) !}{(n / k-j) !}\right)\left(\frac{((k-1) n / k) !}{n !}\right)
\end{aligned}
$$




$$
\begin{aligned}
& \times\left(\frac{((k-1) n / k) !}{((k-2) n / k+j) !}\right) \\
& =\frac{\left(\begin{array}{c}
(k-1) n / k \\
n / k
\end{array}\right)}{\left(\begin{array}{c}
n \\
n / k
\end{array}\right)}+\sum_{j=1}^{\delta n}\left(\begin{array}{c}
n / k \\
j
\end{array}\right) \frac{(n / k)_{j}\left(\frac{(k-1) n}{k}\right)_{n / k-j}}{(n)_{n / k}} \\
& =\frac{\left(\begin{array}{c}
(k-1) n / k \\
n / k
\end{array}\right)}{\left(\begin{array}{c}
n \\
n / k
\end{array}\right)}+\sum_{j=1}^{\delta n}\left(\begin{array}{c}
n / k \\
j
\end{array}\right) \frac{(n / k)_{j}\left(\frac{(k-1) n}{k}\right)_{n / k-j}}{\left(\frac{(k-1) n}{k}+j\right)_{j}(n)_{n / k-j}} \\
& \leq \frac{\left(\frac{(k-1) n}{k}\right)_{n / k}}{(n)_{n / k}}+\sum_{j=1}^{\delta n}\left(\begin{array}{c}
n / k \\
j
\end{array}\right)\left(\frac{1}{(k-1)}\right)^{j}\left(\frac{k-1}{k}\right)^{n / k-j} \\
& \leq\left(\frac{k-1}{k}\right)^{n / k}+\sum_{j=1}^{\delta n}\left(\begin{array}{c}
n / k \\
j
\end{array}\right)\left(\frac{k-1}{k}\right)^{n / k-j} \quad \text { since } 1 /(k-1)<1 \\
& \leq\left(\frac{k-1}{k}\right)^{n / k}+\delta n\left(\begin{array}{c}
n / k \\
\delta n
\end{array}\right)\left(\frac{k-1}{k}\right)^{n / k-\delta n} \quad \text { for } \delta<1 / 2 k \\
& \leq\left(\frac{k-1}{k}\right)^{n / k}+\delta n\left(\frac{e}{k \delta}\right)^{\delta n}\left(\frac{k-1}{k}\right)^{n / k-\delta n} \\
& =\left(1+\delta n\left(\frac{e}{(k-1) \delta}\right)^{\delta n}\right)\left(\frac{k-1}{k}\right)^{n / k} \\
& \leq 2 \delta n\left[\left(\frac{e}{(k-1) \delta}\right)^{\delta}\left(\frac{k-1}{k}\right)^{1 / k}\right]^{n} \quad \text { for } n \text { sufficiently large. }
\end{aligned}
$$

The claim follows after multiplication by $k$.

The following technical lemma allows us to disregard the leading term.

Lemma 3.5. Given $a, t, c>0$, there exists an $N$ such that for $n \geq N$,

$$
c n a^{n}<(a+t)^{n} .
$$

Proof. Note that for $n$ sufficiently large, we have

$$
\log (c n) / n+\log a<\log (a+t) .
$$

Multiplying by $n$ and exponentiating both sides yields the desired result.

The upper bound in Theorem 1.4 is found by combining Lemma 3.4 with Lemma 3.5. 
Next, we find a lower bound for the number of permutations with a low density of a given pattern. In order to make the arguments more natural, we change our notion of low density, from fewer than $\delta^{k} n^{k}$ copies of a given pattern of length $k$, to fewer than $\delta n^{k}$ copies. A simple substitution can then be used to combine our results in the form of Theorem 1.4. We will create permutations that have a low density of a given pattern by using skew sums and direct sums of permutations.

Definition 3.6. Let $\pi \in S_{m}$ and $\sigma \in S_{n}$. The skew sum $\pi \ominus \sigma$ and direct sum $\pi \oplus \sigma$ are permutations in $S_{n+m}$ defined as follows:

$$
\begin{aligned}
& (\pi \ominus \sigma)(i)= \begin{cases}\pi(i)+n & \text { for } 1 \leq i \leq m, \\
\sigma(i-m) & \text { for } m+1 \leq i \leq m+n,\end{cases} \\
& (\pi \oplus \sigma)(i)= \begin{cases}\pi(i) & \text { for } 1 \leq i \leq m, \\
\sigma(i-m)+m & \text { for } m+1 \leq i \leq m+n .\end{cases}
\end{aligned}
$$

For example, let $\pi=2314$ and $\sigma=123$, then $\pi \ominus \sigma=5647123$ and $\pi \oplus \sigma=2314567$.

Note that both the skew and symmetric sums are associative, so the permutations $\bigoplus_{i=1}^{M} \sigma_{i}=\sigma_{1} \oplus \ldots \oplus \sigma_{M}$ and $\ominus_{i=1}^{M} \sigma_{i}=\sigma_{1} \ominus \ldots \ominus \sigma_{M}$ are well-defined.

Theorem 3.7. Given $n$ sufficiently large, $0<\delta<1$, and a pattern $\gamma$ of length $k$, the number of permutations in $S_{n}$ with fewer than $\delta n^{k}$ instances of $\gamma$ is bounded below by $n !\left(\frac{\delta}{2}\right)^{n}$.

Proof. We will use either the direct or skew sum (depending on $\gamma$ ) to create a permutation $\sigma$ with fewer than $\delta n^{k}$ instances of $\gamma$. Let $M=2 / \delta$. As above, we make the assumption that $M$ is an integer that divides $n$, in order to simplify the calculations.

Given $\gamma \in S_{k}$, it must be true that either $\gamma(1)>\gamma(2)$ or $\gamma(1)<\gamma(2)$. In the case of the former, let $\sigma \in S_{n}$ be given by $\sigma=\bigoplus_{i=1}^{M} \sigma_{i}$ where $\sigma_{i} \in S_{n / M}$ for $1 \leq i \leq M$. In the latter case, let $\sigma=\ominus_{i=1}^{M} \sigma_{i}$. For any instance $\sigma(z)$ of $\gamma$, it must be true that $j n / M<z(1)<z(2) \leq(j+1) n / M$, for some $0 \leq j \leq M-1$. Otherwise, the construction forces $\sigma(z(1))<\sigma(z(2))$ in the case of the former, and $\sigma(z(1))>\sigma(z(2))$ in the case of the latter, giving a contradiction. Therefore, the number of instances of $\gamma$ in $\sigma$ is bounded above by:

$$
M\left(\begin{array}{c}
n / M \\
2
\end{array}\right)\left(\begin{array}{c}
n \\
k-2
\end{array}\right)<\frac{n^{k}}{M}<\delta n^{k} \quad \text { by our choice of } M .
$$


Furthermore, the total number of permutations that can be formed using this construction is given by:

$$
\begin{aligned}
{[(n / M) !]^{M} } & \geq(2 \pi n / M)^{M / 2}\left[\frac{(n / M)^{(n / M)}}{e^{(n / M)}}\right]^{M} \quad \text { by Stirling's Approximation } \\
& \geq e \sqrt{n}\left(\frac{n}{e}\right)^{n} M^{-n} \quad \text { for } n \text { sufficiently large } \\
& \geq n !\left(\frac{\delta}{2}\right)^{n}
\end{aligned}
$$

which proves the claim.

\section{Removal lemma}

Next, we use the block partitions of the permutation matrices to prove a pattern removal lemma for permutations. The following definition allows us to make our argument structurally similar to the the proof of the graph removal lemma.

Definition 4.1. The set of edges between blocks $B_{1}$ and $B_{2}$, denoted by $E\left(B_{1}, B_{2}\right)$, is given by

$$
E\left(B_{1}, B_{2}\right)=\left\{\left(\sigma_{i}, \sigma_{j}\right) \mid \sigma_{i} \in B_{1} \text { and } \sigma_{j} \in B_{2}\right\} .
$$

Proof of Theorem 1.8. Let $\sigma$ be $\epsilon$-far from avoiding $\gamma$. As above, assume $n$ is divisible by $\sqrt{m}, m$ to be defined later, and form the block partition $M_{\sigma}^{\sqrt{m}}$. We construct a set $E$ of edges as follows. Let $E \subseteq E(\sigma)$ contain all edges of the following types:

1. edges between blocks that share a row or column (corresponding to the irregular pairs),

2. edges between pairs of blocks in which the order of one block is at most $\frac{n}{m^{2}}$ (corresponding to pairs of low density).

Each column or row of blocks contains exactly $\frac{n}{\sqrt{m}} 1$ 's. Thus, the total number of edges between pairs that share the same row or column is

$$
2 \sqrt{m}\left(\begin{array}{c}
\frac{n}{\sqrt{m}} \\
2
\end{array}\right)<\frac{n^{2}}{2 m}(2 \sqrt{m})=\frac{n^{2}}{\sqrt{m}} .
$$

To count the number of edges of the second type, note that there are $m$ blocks in total, each of which has order at most $\frac{n}{\sqrt{m}}$. Thus, the total number of edges between pairs with this property cannot exceed 


$$
m^{2}\left(\frac{n}{m^{2}}\right)\left(\frac{n}{\sqrt{m}}\right)=\frac{n^{2}}{\sqrt{m}} .
$$

Note that $|E|<\frac{2 n^{2}}{\sqrt{m}}$ edges, so a choice of $m=\frac{4}{\epsilon^{2}}$ ensures that this number is less than $\epsilon n^{2}$. Since $\sigma$ is $\epsilon$-far from avoiding $\gamma, E$ does not cover $J_{\gamma}(\sigma)$, so there exists $s \in J_{\gamma}(\sigma)$ such that $E(s) \cap E=\emptyset$. Note that each edge $(s(i), s(j))$ occurs between pairs of blocks that each have order at least $n / m^{2}=\frac{\epsilon^{4} n}{16}$ and do not share a row or column; so Lemma 3.2 ensures that there are at least

$$
\left(\frac{\epsilon^{4} n}{16}\right)^{k}
$$

instances of $\gamma$ in $\sigma$.

The contrapositive is more recognizable as a removal lemma.

Theorem 4.2. Let $\gamma$ be a pattern of length $k$. If $\sigma$ contains fewer than $\left(\frac{\epsilon^{4} n}{16}\right)^{k}$ instances of $\gamma$, then $d(\sigma, \gamma)<\epsilon$.

\section{Conclusion}

There is a large margin between the two bounds that we have found. In our bound of the form $\left(a^{n}\right) n ! \leq \chi_{\delta}^{n}(\gamma) \leq\left(b^{n}\right) n$ !, a tends to zero as $\delta \rightarrow 0$, but $b$ tends to a constant (less than one), dependent on $k$, the length of the pattern. We think that the actual value of $\chi_{\delta}^{n}(\gamma)$ lies closer to the lower bound that we have found. The proof of the upper bound only relies on the fact that a certain region of the permutation matrix must be relatively sparse, but in the lower bound, the use of skew and direct sums puts a tight restriction on allowable elements in all parts of the permutation, which we believe is more aligned with the correct regime. It should be noted that our bounds only depend on the length $k$ of the permutation $\gamma$, while $\chi_{\delta}^{n}(\gamma)$ likely depends on the structure of $\gamma$ as well, as observed when considering exclusively pattern avoiding permutations. Indeed, $\left|S_{n}(1324)\right|>\left|S_{n}(1234)\right|$ [2] and even $\left|S_{n}(1324)\right|^{1 / n}$ and $\left|S_{n}(1234)\right|^{1 / n}$ are not asymptotically equal.

We believe the block partitions of the permutation matrix will also aid in understanding the "shape" of permutations with a low density of a given pattern, as certain blocks with large order can force large regions of the permutation matrix to be sparse, in order to contain a low density of a given pattern.

\section{Acknowledgments}

The author would like to thank Eric Babson for suggesting the problem, and for many years of invaluable guidance and support. He also thanks 
Jesse and Laura for help in the editing process, and the referees for their valuable feedback and suggestions.

\section{References}

[1] N. Alon and J. Spencer, The Probabilistic Method, Third Edition, Wiley Interscience Series in Discrete Math. and Optimization, 2008, 289-302. MR2437651

[2] M. Bóna, Combinatorics of Permutations, Chapman \& Hall/CRC, 2004, 129-205. MR2078910

[3] J. Cooper, A permutation regularity lemma, Electr. J. Comb., 2006, 13(1), R22. MR2212495

[4] J. Cooper, E. Lundberg, and B. Nagle, Generalized pattern frequency in large permutations, Electr. J. Comb., 2013, 20(1), P28. MR3035038

[5] R. Diestel, Graph Theory, Springer, 1991, 147-169. MR2744811

[6] B. Green and T. Tao, The primes contain arbitrarily long arithmetic progressions, Annals of Mathematics, 2008, 167(2), 481-547. MR2415379

[7] W. Gowers, Lower bounds of tower type for Szemerédi's uniformity lemma, Geometric and Functional Analysis, 1997, 7(2), 322-337. MR1445389

[8] S. Kitaev, Patterns in Permutations and Words, Springer Verlag, 2011. MR3012380

[9] A. Marcus and G. Tardos, Excluded permutation matrices and the Stanley-Wilf conjecture, J. Combin. Theory Ser. A, 2004, 107(1), 153160. MR2063960

[10] E. Steingrímsson, Some open problems on permutation patterns, London Mathematical Society Lecture Note Series, 2013, 239-263. MR3156932

[11] E. Szemerédi, On sets of integers containing no $k$ elements in arithmetic progression, Acta Arithmetica, 1975, 27, 199-245. MR0369312

[12] T. Tao, A variant of the hypergraph removal lemma, J. Comb. Theory, Ser. A, 2006, 113(7), 1257-1280. MR2259060 
BENJAMIN FINEMAN

310 12TH ST

DAVIS, CA 95616

USA

E-mail address: fineman@math.ucdavis.edu

ReCEIVEd 12 February 2014 